\title{
Il trattamento topico con una matrice contenente mesoglicano in associazione ad acido ialuronico nella gestione delle ulcere cutanee croniche degli arti inferiori
}

\author{
Rolando Tasinato, ${ }^{1}$ Paolo Zangrande ${ }^{2}$ \\ ${ }^{1}$ Unità Operativa di Chirurgia Generale, ASL 3 Serenissima, Ospedale di Mirano (VE); ${ }^{2}$ Unità Operativa di Pronto Soccorso, ASL 3 \\ Serenissima, Ospedale di Mirano (VE), Italia
}

\section{RIASSUNTO}

Un'ulcera cutanea cronica degli arti inferiori rappresenta l'epifenomeno di varie situazioni patologiche che possono coinvolgere, a vari livelli e con diversi gradi di interessamento, i sistemi venoso, arterioso e linfatico della gamba. Recentemente sono state pubblicate numerose evidenze secondo cui i glicosaminoglicani (GAG) svolgono un ruolo importante nella riepitelizzazione delle ulcere cutanee croniche degli arti inferiori, in particolare per quanto riguarda le ulcere ad eziopatogenesi vascolare venosa o mista. In questo articolo riportiamo la nostra esperienza nel trattamento delle ulcere cutanee croniche degli arti inferiori con una metodica che prevede il debridement secondo il protocollo TIME, antisepsi con una soluzione di iodopovidone al $10 \%$ la successiva l'applicazione di una medicazione bioattiva contenente GAG e acido ialuronico. Questa metodica è stata confrontata con un'analoga metodica che prevede sempre lo stesso tipo di debridement e di antisepsi con soluzione di iodopovidone al $10 \%$ affiancata a una medicazione non bioattiva.

\section{INTRODUZIONE}

Le ulcere cutanee croniche degli arti inferiori sono costituite da un gruppo variegato di situazioni patologiche dove molteplici sono i fattori eziopatogenetici che incidono e condizionano radicalmente le strategie di trattamento. Tale trattamento deve essere necessariamente diversificato in base alla patologia di base che sostiene l'evento ulcera-

Corrispondenza: Rolando Tasinato, Unità Operativa di Chirurgia Generale, ASL 3 Serenissima, Ospedale di Mirano, Via Don Giuseppe Sartor 4, 30035 Mirano (VE), Italy.

E-mail: rolandotasinato@libero.it

Parole chiave: Ulcere cutanee croniche; Mesoglicano; Acido ialuronico; Debridement.

Contributi: RT: medico specialista in chirurgia generale, perfezionato in angiologia e flebologia, vulnologia e trattamento del piede diabetico; PZ: medico specialista in chirurgia generale, diagnostica vascolare non invasiva e vulnologia.

Conflitti d'interesse: gli autori dichiarano l'assenza di conflitti d'interesse.

Fondi: nessuno.

Ricevuto per la pubblicazione: 7 febbraio 2019.

Revisione ricevuta: 6 marzo 2019.

Accettato per la pubblicazione: 15 maggio 2019 .

This work is licensed under a Creative Commons Attribution NonCommercial 4.0 License (CC BY-NC 4.0).

CCopyright R. Tasinato e P. Zangrande, 2019

Licensee PAGEPress, Italy

Italian Journal of Wound Care 2019; 3(2):49-53

doi:10.4081/ijwc.2019.48 tivo anche se alcuni principi restano validi per un approccio condiviso alle varie tipologie di lesione. ${ }^{1,2}$

Un'ulcera cutanea cronica a livello degli arti inferiori rappresenta pertanto l'epifenomeno di patologie che possono coinvolgere, a vario livello il sistema venoso, arterioso, linfatico e nervoso della gamba che vanno identificate e adeguatamente trattate.

Strategie diversificate che, tuttavia, concordano nel fatto che un accurato debridement associato alla detersione e disinfezione del fondo dell'ulcera e della cute perilesionale sono presupposti indispensabili per la promozione di una adeguata riepitelizzazione, nell'ottica precipua di una riduzione dei tempi di guarigione.

I risvolti in termini socio-sanitari e le rilevanti implicazioni economiche connesse a queste patologie ed alla gestione di questi pazienti sono di comune riscontro in quanto il problema delle lesioni ulcerative croniche incide pesantemente nel budget annuale delle aziende socio sanitarie. ${ }^{3}$

Se da una parte molti trials multicentrici randomizzati hanno evidenziato che il trattamento antibatterico, topico o sistemico, di fatto non influenza le modalità ed i tempi di guarigione ${ }^{4}$ in anni più recenti esperienze cliniche multicentriche evidenziano un sensibile vantaggio nel trattamento antibatterico topico di queste lesioni.

Recentemente sono sempre maggiori le evidenze che attribuiscono ai glicosaminoglicani (GAG) un ruolo favorente la riepitelizzazione delle lesioni ulcerative cutanee croniche degli arti inferiori in particolare per quanto riguarda le ulcere di origine vascolare venosa e arteriosa.

In questo lavoro riportiamo la nostra esperienza relativa al trattamento delle ulcere cutanee croniche con debridement secondo il protocollo TIME Wound Bed Preparation e applicazione di una medicazione bioattiva contenente acido ialuronico e GAG rispetto ad una meto- 
dica con medicazione standard che prevede la detersione delle lesioni con soluzione di iodopovidone al 10\%.

\section{MATERIALI E METODI}

Dal gennaio 2017 al aprile 2017 presso l'ambulatorio Vulnologico di Chirurgia Vascolare e di Chirurgia Generale del Presidio Ospedaliero ASL 3 Serenissima di Mirano (Venezia) sono stati trattati consecutivamente 63 pazienti riconosciuti affetti da ulcere cutanee croniche localizzate agli arti inferiori.

Si tratta di 29 uomini e 34 donne di età media 68,1 anni (range 32-91) inviati e valutati presso il nostro ambulatorio vulnologico allestito nell'ambito di una unità operativa di chirurgia generale con annessa sezione di chirurgia vascolare ed emodinamica interventistica.

I pazienti erano stati selezionati dopo adeguata valutazione clinica, studio ecocolordopler, in molti casi anche implementato da angioTAC o angiografia, e valutazione dell'ossimetria transcutanea.

Questo al fine di formulare una corretta diagnosi per poi successivamente avviare il paziente a trattamento specifico della patologia sottostante che riassumiamo in Tabella 1.

Una volta preso in carico il paziente, la lesione veniva fotografata e sottoposta a misurazione mediante tratteggio dei margini su foglio trasparente di acrilato e stima dell'area con valutazione centimetrica (Figura 1).

Le impronte così ottenute potevano essere catalogate e paragonate nei controlli successivi ottenendo una rapida visualizzazione degli eventuali progressi in termini di riduzione o meno dell'area nel corso del trattamento.

Nell'ambito di uno studio più generale sulle ulcere che giungono alla nostra osservazione in ambulatorio, abbiamo effettuato una randomizzazione dei 53 pazienti i due gruppi A e B diversificati in base alla tipologia di trattamento topico della lesione diversificando i pazienti.

Nel gruppo A abbiamo adottato una metodica standard: detersione della lesione ulcerativa con soluzione fisiologica per contatto di 5 minuti, debridement del letto con rimozione meccanica del biofilm mediante detersione con garza e successiva duplice applicazione di iodopovidonee al $10 \%$ sia del letto ulcerativo che della cute perilesionale per una distanza di almeno $5 \mathrm{~cm}$ dal margine della lesione, Seguiva medicazione occlusiva a piatto associata a bendaggio elastico nel caso di ulcera flebostatica.

I pazienti del gruppo B sono stati trattati similmente ma dopo la detersione e disinfezione abbiamo utilizzato una medicazione bioattiva (matrice contenente biofilm a base di mesoglicano in associazione con acido ialuronico).

La matrice che contiene il biofilm presenta un effetto duplice: i GAG, come componenti della matrice extracellulare, favoriscono la risposta delle citochine, responsabili del meccanismo di ricostruzione del tessuto lesionato. Inoltre, il biofilm agisce anche formando una barriera protettiva sulla lesione e contribuisce a mantenere un microambiente favorevole al processo di cicatrizzazione.

Anche in questo gruppo i pazienti venivano trattati con: detersione della lesione ulcerativa con soluzione fisiologica per contatto per circa 5 minuti, debridement del letto con rimozione meccanica del biofilm mediante detersione con garza impregnata e successiva applicazione della matrice con biofilm contenente mesoglicano sul letto ulcerativo con tempo di contatto di almeno 3 minuti prima di procedere alla medicazione occlusiva a piatto o bendaggio elastico nel caso di ulcera flebo statica.

La medicazione veniva praticata ogni 2-3 giorni. Nelle

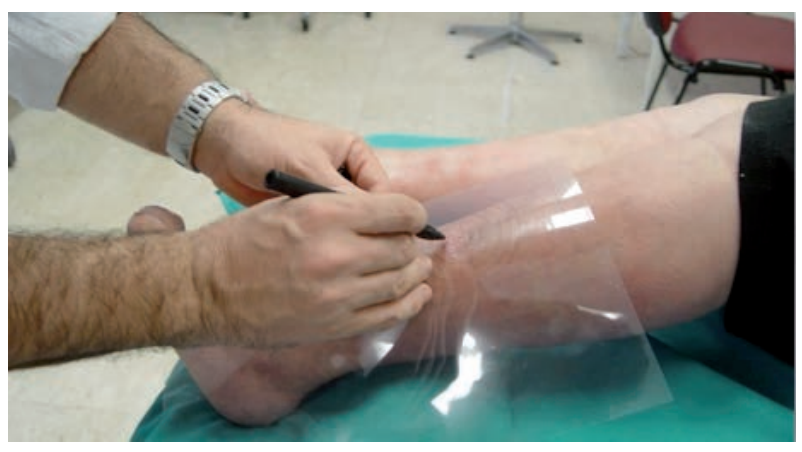

Figura 1. Metodica adottata presso il nostro ambulatorio per la valutazione dimensionale delle lesioni ulcerative.

Tabella 1. Stratificazione dei pazienti in base alla tipologia di ulcera cutanea per la quale sono stati trattati in ambulatorio.

\begin{tabular}{lcccccc} 
Tipo di ulcera & $\mathbf{N}^{\circ}$ totale pazienti & Maschi & Femmine & Media settimane di trattamento & \% casi giunti a riepitelizzazione \\
\hline Flebostatica & 26 & 10 & 16 & 7.2 & 94.8 \\
\hline Ischemica & 17 & 9 & 8 & 9.5 & 79.3 \\
\hline Neuropatica & 8 & 3 & 5 & 5.9 & 92.2 & 96.4 \\
\hline Post-traumatica & 4 & 3 & 1 & 5.6 & 98.8 \\
\hline Ustione & 2 & 1 & 1 & 3.5 & 74.2 \\
\hline Vasculitica & 2 & 0 & 2 & 11.4 & 88.6 \\
\hline Mista & 4 & 2 & 2 & 9.1 &
\end{tabular}


fasi iniziali, specie nel caso di lesioni molto secernenti, anche ogni due giorni rinnovando la medicazione in base all'entità della secrezione; mediamente da 1 a 3 volte la settimana a seconda dei casi. Diversamente nelle fasi finali, a riepitelizzazione quasi ultimata, la medicazione veniva sostituita anche dopo 4-5 giorni.

Gli accessi e l'andamento clinico dei pazienti venivano di volta in volta registrati in un apposito diario clinico digitalizzato corredando la documentazione anche di immagini fotografiche.

La gestione complessiva dei pazienti è stata effettuata anche con l'ausilio del personale A.D.I (assistenza infermieristica domiciliare) che provvedeva all'applicazione del protocollo a domicilio specie nei pazienti difficilmente trasportabili riportando in cartella clinica i dati.

\section{RISULTATI}

Dei 65 pazienti iniziali $63(93,6 \%)$ hanno portato a termine la randomizzazione collezionando 32 pazienti nel gruppo A e 31 pazienti nel gruppo B.

Complessivamente, in entrambi i gruppi randomizzati il trattamento è stato ben tollerato.

Nel gruppo A abbiamo osservato in 2 casi $(6,3 \%)$ fenomeni di ipersensibilizzazione cutanea legata alla presenza del composto iodato o degli eccipienti in esso contenuti.

Nel gruppo B solo in 1 caso $(3,2 \%)$ abbiamo osservato reazioni cutanee di tipo allergico/eritematoso che ci hanno indotto alla sospensione del trattamento che complessivamente, nel $96,8 \%$ dei casi è stato ben tollerato.

Per quanto riguarda la sintomatologia dolorosa tutti i pazienti randomizzati sono stati valutati a ogni accesso mediante la numeric rating scale (NRS) ad 11 items per la misurazione del dolore.

Nella Figura 2 riportiamo i rilevamenti medi della sintomatologia dolorosa nei due gruppi di pazienti. L'analisi evidenzia un andamento pressoché sovrapponibile per quanto riguarda durata e intensità del dolore.

\section{DISCUSSIONE}

Sottolineiamo il rilievo di una riduzione della sintomatologia dolorosa media nel gruppo di pazienti sottoposti a medicazione topica con biofilm contenente mesoglicano ed acido ialuronico riduzione del dolore valutabile intorno a 2,5 item NRS rispetto al gruppo di controllo con medicazione standard.

La riduzione del dolore si apprezza in particolare nelle ore successive alla seduta di medicazione Tale effetto ha comportato una riduzione del $21,6 \%$ nell'assunzione di analgesici per os nei pazienti del gruppo B rispetto al gruppo A.
Per quanto concerne la velocità di riepitelizzazione e conseguentemente i tempi di guarigione delle lesioni, pur nell'eterogeneità della tipologia di lesioni abbiano osservato un miglioramento dei tempi di guarigione nel gruppo di pazienti trattati con biofilm contenente mesoglicano/ acido ialuronico rispetto al gruppo di pazienti trattati con metodica standard.

Nel caso di ulcere classificate come flebostatiche (32 pazienti consecutivi) il tempo di riepitelizzazione nel gruppo B era mediamente di 11,9 giorni inferiore rispetto al gruppo dei pazienti trattati con composto iodato (Tabella 2).

Anche per le altre tipologie di ulcere il tempo medio di riepitelizzazione del gruppo $\mathrm{B}$ era inferiore anche se in percentuale minore tale da non consentire per il momento di estrapolare dati statisticamente significativi come per il caso delle ulcere flebo statiche, gruppo numericamente più consistente nella nostra casistica di valutazione preliminare.

Sotto il profilo batteriologico abbiamo osservato un'efficacia sostanzialmente sovrapponibile delle due modalità di medicazione riferendosi al povidone iodato nell'azione antisettica.

Il numero dei casi in cui abbiamo osservato comparsa di febbre o sovra infezione batterica è stato sostanzialmente sovrapponibile nei due gruppi (Tabella 3).

Più interessante appare l'analisi dei dati emersi dalle indagini colturali effettuate su tampone diretto del fondo della lesione, che hanno tuttavia evidenziato che dopo la 4 settimana di trattamento aumentano le lesioni contaminate da miceti, forme microbiche che sono scarsamente sensibili all'azione dei composti iodati.

La valutazione di altri parametri clinici; in particolare la presenza di sintomatologia dolorosa notturna, il conseguente ricorso ad antidolorifici e il numero di accessi per

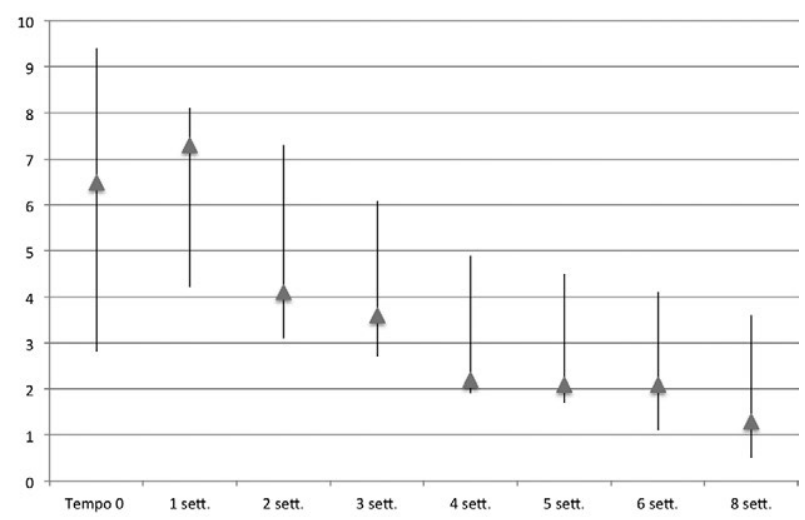

Figura 2. Rilievi medi della sintomatologia dolorosa rilevata con misurazione NRS a 11 items in occasione delle valutazioni ambulatoriali dei 63 pazienti complessivamente randomizzati nei due gruppi. 
medicazioni in ambulatorio, nei due gruppi di pazienti, ha documentato come i pazienti del gruppo B (medicazione topica con biofilm a base di mesoglicano e acido ialuronico) manifestano meno sintomatologia dolorosa in particolare dopo la seduta di medicazione rispetto ai pazienti trattati nel gruppo A (Figura 3).

\section{CONCLUSIONI}

Dal punto di vista gestionale, la detersione e l'applicazione topica una matrice bioattiva contenente mesoglicano in associazione ad acido ialuronico si è rilevata efficace sia sotto il profilo clinico che gestionale in relazione alla sicurezza ed alla facilità di impiego. ${ }^{5-7}$

In particolare, l'introduzione della medicazione con matrice bioattiva (Figura 4) contenente mesoglicano non ha modificato o interferito in modo sostanziale con le altre procedure e le linee guida che da tempo abbiamo adottato nel nostro Ospedale per la gestione dei pazienti con ulcera cronica degli arti inferiori. La medicazione con matrice attiva con mesoglicano in particolare si integra in modo molto efficace con il protocollo TIME Wound Bed Prepa- ration che da circa 3 anni abbiamo adottato e reso comune tra i vari servizi e centri interaziendali che si occupano della gestione di questi pazienti. ${ }^{8}$

In particolare, abbiamo notato che la solubilizzazione

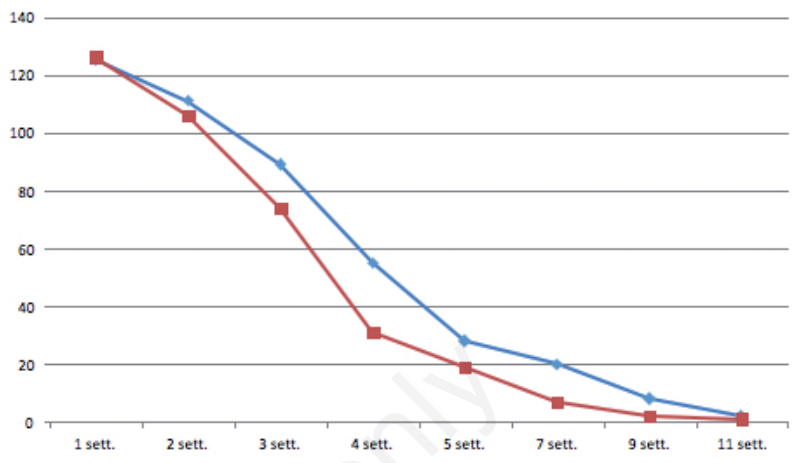

Figura 3. Valutazione comparativa dell'andamento della riepitelizzazione delle ulcere nei due gruppi randomizzati. Gruppo A (linea rossa): pazienti trattati con polivinilpirrolidone iodato. Gruppo B (linea azzurra): pazienti trattati con matrice bioattiva contenente mesoglicano in associazione ad acido ialuronico.

Tabella 2. Rilievi clinici e calcolo dei tempi di riepitelizzazione media nei due gruppi di pazienti oggetto dello studio.

\begin{tabular}{lrr} 
& $\begin{array}{c}\text { Gruppo A - Trattamento con } \\
\text { polivinilpirrolidone iodato }\end{array}$ & $\begin{array}{c}\text { Gruppo B - Trattamento con } \\
\text { matrice bioattiva Prismaskin }\end{array}$ \\
$\begin{array}{l}\text { Tempo di riepitelizzazione complessivo in giorni dal primo accesso in ambulatorio } \\
\text { Febbre }\end{array}$ & 42,4 giorni & 31,6 giorni \\
\hline Dolore maggiore di 5 NRS & 2 casi & 1 caso \\
\hline Ricorso ad antidolorifici & 18 casi & 2 casi \\
\hline Intolleranza alla procedura di medicazione & 2 casi & 5 casi \\
\hline Pazienti che hanno necessitato di più di 3 accessi settimanali in ambulatorio & 13 casi & 1 caso \\
\hline Numero di casi non riepitelizzati dopo 15 settimane di trattamento & 5 casi & 4 casi \\
\hline
\end{tabular}

NRS, numeric rating scale.

Tabella 3. Ceppi microbici isolati al tempo zero (data di accesso in ambulatorio) e dopo 4 settimane di trattamento nei due gruppi di pazienti. Si nota come dopo 4 settimane inizia a essere significativamente presente la quota di forme micotiche. Questa evenienza si è dimostrata particolarmente frequente nei pazienti che erano stati trattati con antibioticoterapia topica o sistemica.

\begin{tabular}{|c|c|c|c|}
\hline \multicolumn{2}{|c|}{ Frequenza ceppi microbici isolati al tempo zero } & \multicolumn{2}{|c|}{ Frequenza ceppi microbici isolati dopo 4 settimane di trattamento } \\
\hline Pseudomonas Aeruginosa & $* * * *$ & Fusobacterium & $* * * *$ \\
\hline Staphylococcus Aureus & $* * *$ & Streptococcus Epidermidis & $* *$ \\
\hline Altri Staphylococci & $* *$ & Peptostreptococci & $* *$ \\
\hline Proteus Mirabilis & $* * *$ & Klebsiella Sporigens & $* *$ \\
\hline Escherichia Cloacae & $* *$ & Acinetobacter & $* *$ \\
\hline Enterococcus Fecalis & $* *$ & Escherichia Coli & $* *$ \\
\hline Clostridium Sporigens & * & Candida Sporigens & $* *$ \\
\hline Candida Sporigens & $* *$ & Epidermophyton & $* *$ \\
\hline
\end{tabular}

$*, * *, * * *, * * * *$ gli asterischi rappresentano la frequenza relativa dei ceppi microbici rinvenuti (da uno a quattro) in ordine crescente. 


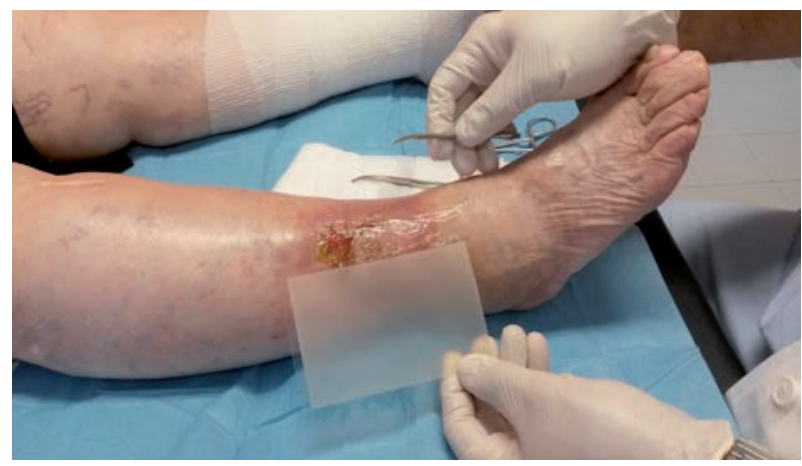

Figura 4. Esemplificazione della medicazione con membrana bioattiva contenente mesoglicano in associazione con acido ialuronico. La membrana viene appoggiata sopra la lesione ulcerativa direttamente a contatto con il fondo dell'ulcera. Successivamente, il paziente viene trattato con una medicazione occlusiva semplice associata o meno a bendaggio elastocompressivo in base alle linee guida previste dal il tipo specifico di lesione.

della matrice non lascia depositi o residui evidenti sul letto dell'ulcera consentendo in questo senso una valutazione diretta del fondo dell'ulcera e non richiede ulteriori manovre di debridement se non quelle tese alla rimozione dello sludge eventualmente riformatosi tra una medicazione e l'altra.

Anche dal punto di vista economico tale medicazione si è dimostrata molto più vantaggiosa rispetto alla maggior parte delle opzioni terapeutiche attualmente adottate nel trattamento delle ulcere cutanee croniche.

Anche per quanto riguarda l'addestramento del personale infermieristico, in particolare afferente al Servizio ADI della nostra ASL la membrana bio attiva contenente mesoglicano si è dimostrata di facile ed intuitivo utilizzo richiedendo tempi di apprendimento molto contenuti .

Il notevole contenimento dei costi, l'applicabilità in numerose situazioni cliniche, l'efficace azione pro-riepitelizzante, in particolare per quanto riguarda le ulcere cutanee croniche di origine flebostatica e l'ottimale profilo di sicurezza del prodotto ci ha indotto a inserire Prismaskin matrice bioattiva quale medicazione efficace nel novero delle opzioni terapeutiche per la gestione dei pazienti con ulcere cutanee croniche degli arti inferiori.

\section{BIBLIOGRAFIA}

1. Nelson EA, Ruckley CV, Dale J, Morison M. The management of leg ulcers. J Wound Care 1996;5:73-6.

2. Makrantonaki E, Wlaschek M, Scharffetter-Kochanek K. Pathogenesis of wound healing disorders in the elderly. J Dtsch Dermatol Ges 2017;15:255-75.

3. Humbert P, Mikosinki J, Benchikhi H, Allaert FA. Efficacy and safety of a gauze pad containing hyaluronic acid in treatment of leg ulcers of venousor mixed origin: a double-blind, randomised, controlled trial. Int Wound J 2013;10:159-66.

4. Meaume S, Ourabah Z, Romanelli M, et al. Efficacy and tolerance of a hydrocolloid dressing containing hyaluronic acid for the treatment of leg ulcers of venous or mixed origin. Curr Med Res Opin 2008;24:2729-39.

5. Dissemond J, Böttrich JG, Braunwarth H, et al. Evidence for silver in wound care - meta-analysis of clinical studies from 2000-2015. J Dtsch Dermatol Ges 2017;15:524-35.

6. Frantz RA, Bergquist S, Specht J. The cost of treating pressure ulcers following implementation of a research-based skin care protocol in a long-term-care facility. Adv Wound Care 1995;8:36-45.

7. Gao AL, Cole JG, Stoecker WV. Unna boot central gauze technique for chronic venous leg ulcers. Dermatol Online J 2017;23.

8. Bennett LL, Rosenblum RS, Perlov C, et al. An in vivo comparison of topical agents on wound repair. Plast Reconstr Surg 2001;108:675-87. 